Autorização concedida ao Repositório Institucional da Universidade de Brasília (RIUnB) pelo Professor Kleber Melo e Silva, em 31 de janeiro de 2019, para disponibilizar o trabalho, gratuitamente, para fins de leitura, impressão e/ou download, a título de divulgação da obra. 


\title{
Modified DFT-Based Phasor Estimation Algorithms for Numerical Relaying Applications
}

\author{
K. M. Silva, Member, IEEE, F. A. O. Nascimento, Member, IEEE
}

\begin{abstract}
In this paper, new modifications of the conventional full cycle and half cycle Discrete Fourier Transform (DFT)-based phasor estimation algorithms are proposed. The key idea is to combine two consecutive outputs of their real and imaginary part filters to eliminate the harmful effect of the decaying dc components on phasor estimation, thereby a new strategy to estimate the exponential term is also reported. Proposed algorithms are compared with other DFT-based algorithms by using both computer-simulated and Alternative Transients Program (ATP)generated signals. The obtained results reveal that proposed algorithms present excellent results, even taking into account multiple decaying de components.
\end{abstract}

Index Terms-Phasor estimation, DFT-based algorithms, decaying dc component effect elimination.

\section{INTRODUCTION}

$\mathbf{P}$ HASOR estimation process plays a major role in modern numerical relays [1]. Indeed, in order to correctly estimate phasors, the digital filtering algorithms used in numerical relays must provide bandpass response about the fundamental frequency, good transient behavior, harmonic elimination and decaying dc component rejection as well. Undoubtedly, the last one is harder to deal with, since the decaying dc component is a non-periodic signal whose frequency spectrum encompasses a wide range of frequencies [2]. As a result, the decaying dc component causes a undesired error in phasor estimation outputs, leading protection schemes to misoperate. For example, distance protection either undereaches or overreaches the fault point, whereas overcurrent protection may loss selectivity [3].

Several algorithms to eliminate the decaying dc component effect on phasor estimation have been reported [4]-[19]. Nonetheless, the most widespread are those based on modifications of the traditional full-cycle DFT (FCDFT) and half-cycle DFT (HCDFT) algorithms [11]-[19]. In [11], the parameters of the decaying dc component are estimated using either two successive outputs of the FCDFT algorithm or three successive outputs of the HCDFT algorithm, thereby the dc value at each sampling instant is subtracted from the original samples. However, the performance of these algorithms is severely affected by the analog anti-aliasing filter, because it produces additional decaying dc components as well. Therefore, in order to overcome this drawback, the same authors reported in [12] modified versions of these algorithms, by taking into account the characteristic equation of first- and second-order analog low pass filters. As a consequence, the complexity of these

This research was supported in part by the Brazilian National Council for Scientific and Technological Development (CNPq) and in part by the Brazilian utility Eletrobras Eletronorte.

Kleber M. Silva and Francisco A. O. Nascimento are with the Department of Electrical Engineering at the University of Brasília (UnB), 70910-900 Brasília, Brazil (e-mail: klebermelo@unb.br, assis@unb.br). algorithms increases tremendously. In [13], the output of the FCDFT filters tuned at a frequency far higher than the cutoff frequency of the anti-aliasing filter is used to estimate the dc component parameters, thereby the phasor estimation is corrected. However, since high-frequency components are not completely filtered-out by the anti-aliasing filter, the performance of this algorithm may be jeopardized. In [14], a modification of FCDFT based on partial sums of one cycle data window is reported. Three simplified versions of this algorithm are presented in order to alleviate the computation burden, leading to good results for a wide range of decaying time constants, but do not perform well for the smaller ones. In [15], algebraic manipulations with three successive FCDFT outputs are used recursively to eliminate the decaying dc component effect on phasor estimation, what, in turn, requires high sampling frequency to compensate the inherent time delay. In [16] it is suppressed using the difference between the outputs of the FCDFT for odd and even samples of the full-cycle data window. In [17], the decaying dc component effect is eliminated by combining the FCDFT outputs for odd and even samples obtained trough the decimation of the fullcycle data window by two and by four. The disadvantage of the algorithms reported in [16] and [17] lies in the fact they require the use of additional digital low pass filters to reduce noise sensitivity and to avoid aliasing during decimation process, respectively. The parameters of the decaying dc component are estimated by integrating the full-cycle data window in [18], thereby the output of the FCDFT is corrected. However, by doing so, this algorithm may not be able to properly deal with multiple decaying dc components. In [19], the decaying dc component effect is gotten rid of by combining the outputs of the FCDFT filters for even and odd samples of a signal sampled at a high sampling rate, what shall limit the use of these algorithms in real-world numerical relaying applications.

In this paper, a generalized modification of the conventional DFT-based algorithms is proposed, such that it can be applied to FCDFT and HCDFT algorithms interchangeably. The key idea is to combine two consecutive outputs of their real and imaginary part filters, in order to eliminate the decaying dc component effect. Therefore, a new strategy to estimate the exponential term of the decaying dc component is also reported. Moreover, the proposed fomulation properly deals with variable sampling measurement environment. They are compared with other phasor estimation algorithms by means of computer-simulated and ATP-generated signals as well. The obtained results reveal that proposed DFT-based algorithms provide a simple and efficient mean to perform phasor estimation, even when multiple decaying dc components are taken into account. 


\section{Conventional DFT-Based Algorithms}

The fundamentals of the conventional DFT-based algorithms are briefly presented next.

\section{A. Basic Formulation}

The fundamental frequency phasor $\bar{X}[n]$ of a sinusoidal discrete signal $x[n]$ can be computed using the DFT-based algorithms in their complex form as follows:

$$
\bar{X}[n]=\frac{2}{M} \sum_{m=1}^{M} x[n-M+m] e^{-j m \delta}
$$

where $n$ represents the $n$-th sampling instant; $\delta=\frac{2 \pi}{N} ; N$ is the number of samples per cycle; and $M$ defines the data window length, so that $M=N$ for the FCDFT algorithm and $M=N / 2$ for the HCDFT algorithm [20].

The DFT-based algorithms can be also applied in their rectangular form, i.e., by computing the real part $X_{r e}[n]$ and the imaginary part $X_{i m}[n]$ of $\bar{X}[n]$ separately, as follows:

$$
\begin{gathered}
X_{r e}[n]=\frac{2}{M} \sum_{m=1}^{M} x[n-M+m] \cos (m \delta) \\
X_{i m}[n]=-\frac{2}{M} \sum_{m=1}^{M} x[n-M+m] \sin (m \delta)
\end{gathered}
$$

One can further compute $X_{r e}[n]$ applying the real part filter (i.e., the cosine filter) for both even- and odd-sample-sets [21]:

$$
X_{r e}[n]=\underbrace{X_{r e}^{e v e n}[n]}_{\frac{1}{2} X_{r e}[n]}+\underbrace{X_{r e}^{\text {odd }}[n]}_{\frac{1}{2} X_{r e}[n]}
$$

where $X_{r e}^{\text {even }}[n]$ and $X_{r e}^{\text {odd }}[n]$ are, respectively, the outputs of the real part filter to the even- and odd-sample-sets, which are computed as follows:

$$
\begin{gathered}
X_{r e}^{\text {even }}[n]=\frac{2}{M} \sum_{m=1}^{M / 2} x[n-M+2 m] \cos (2 m \delta) \\
X_{r e}^{\text {odd }}[n]=\frac{2}{M} \sum_{m=1}^{M / 2} x[n-M+2 m-1] \cos [(2 m-1) \delta]
\end{gathered}
$$

Likewise, $X_{i m}[n]$ can be also obtained applying the imaginary part filter (i.e., the sine filter) for both even- and oddsample-sets [21]:

$$
X_{i m}[n]=\underbrace{X_{i m}^{\text {even }}[n]}_{\frac{1}{2} X_{i m}[n]}+\underbrace{X_{i m}^{\text {odd }}[n]}_{\frac{1}{2} X_{i m}[n]}
$$

where $X_{i m}^{\text {even }}[n]$ and $X_{i m}^{\text {odd }}[n]$ are, respectively, the outputs of the imaginary part filter to the even- and odd-sample-sets, which are computed as follows:

$$
\begin{gathered}
X_{i m}^{\text {even }}[n]=-\frac{2}{M} \sum_{m=1}^{M / 2} x[n-M+2 m] \sin (2 m \delta) \\
X_{i m}^{\text {odd }}[n]=-\frac{2}{M} \sum_{m=1}^{M / 2} x[n-M+2 m-1] \sin [(2 m-1) \delta]
\end{gathered}
$$

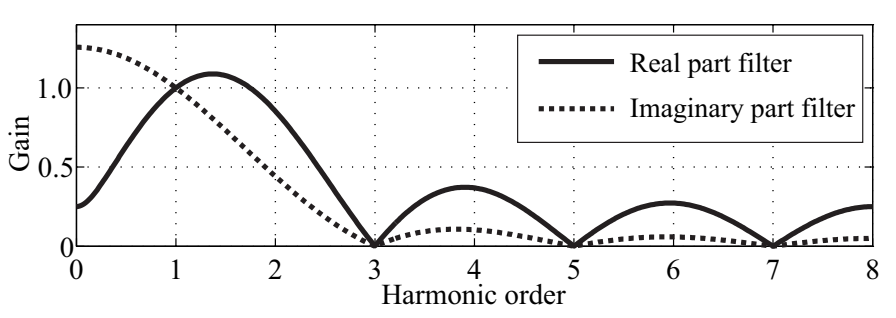

(a)

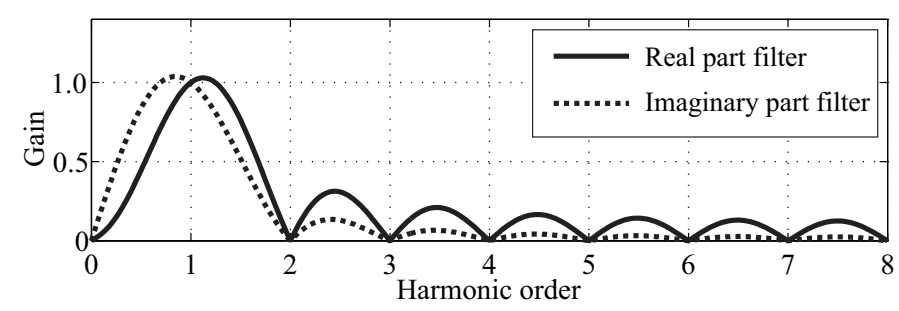

(b)

Fig. 1. Frequency response magnitudes: (a) HCDFT and (b) FCDFT filters.

\section{B. Frequency Response}

The frequency response magnitudes of the HCDFT and FCDFT filters are shown in Fig. 1(a) and 1(b), respectively. They are computed using a sampling rate of 16 samples per cycle. It is observed that the FCDFT filters have total harmonic rejection. Conversely, the characteristics of the HCDFT filters are worse, especially in respect of dc component and even harmonics no-rejection. Also, the frequency response of the HCDFT filters have larger side-lobes, leading to higher sensitivity to interharmonic components [1], [2].

\section{Decaying DC Component Effect}

Consider a discrete signal $x[n]$ described as follows:

$$
x[n]=A_{0} \Gamma^{n}+A_{1} \cos \left(n \delta+\varphi_{1}\right)=x_{d c}[n]+x_{1}[n]
$$

in which $\Gamma=e^{-\Delta t / \tau}$ is the exponential term; $A_{0}$ and $\tau$ are the amplitude and the time constant of the decaying dc component; $A_{1}$ and $\varphi_{1}$ are the amplitude and phase angle of the fundamental frequency phasor; $\Delta t$ is the sampling interval.

Substituting (10) in (1) yields:

$$
\bar{X}[n]=\bar{X}_{d c}[n]+\bar{X}_{1}[n],
$$

where $\bar{X}_{d c}[n]$ and $\bar{X}_{1}[n]$ are the outputs of the DFT-based algorithms to the decaying dc and pure cos-sinusoidal components, which are computed as follows:

$$
\begin{gathered}
\bar{X}_{d c}[n]=\frac{2}{M} \sum_{m=1}^{M} x_{d c}[n-M+m] e^{-j m \delta} \\
=\frac{2}{M} \sum_{m=1}^{M} A_{0} \Gamma^{n-M+m} e^{-j m \delta} \\
\bar{X}_{1}[n]=\frac{2}{M} \sum_{m=1}^{M} x_{1}[n-M+m] e^{-j m \delta} \\
=\frac{2}{M} \sum_{m=1}^{M} A_{1} \cos \left[(n-M+m) \delta+\varphi_{1}\right] e^{-j m \delta}
\end{gathered}
$$




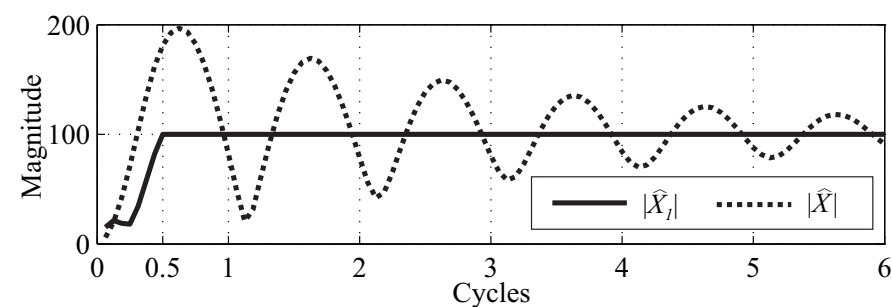

(a)

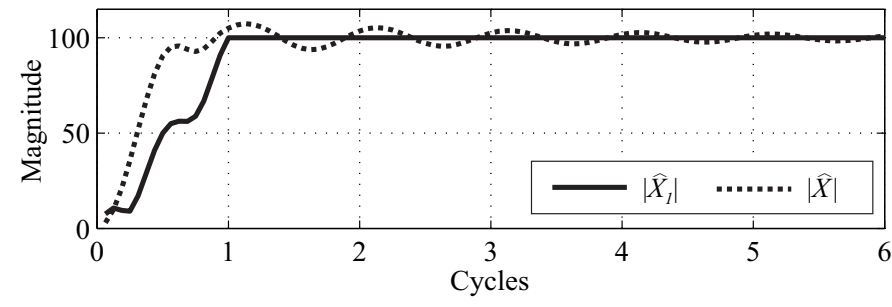

(b)

Fig. 2. The decaying dc component effect on the DFT-based algorithms: (a) HCDFT and (b) FCDFT.

Analyzing (13), one can see that $\bar{X}_{1}[n]$ is the desired fundamental frequency phasor:

$$
\bar{X}_{1}[n]=A_{1} e^{j \varphi_{1}+n \delta} .
$$

Solving (12) yields:

$$
\bar{X}_{d c}[n]=\frac{2}{M} A_{0} \Gamma^{n-M} \frac{\left(1-\Gamma^{M}\right)}{1-\Gamma e^{-j \delta}}, \quad n \geq M .
$$

It can be seen from (11)-(15) that the DFT-based algorithms lead to an error in the fundamental frequency phasor estimation due to the decaying dc component effect. As a result, undesirable oscillations about the actual value of the phasor take place. Aiming to exemplify this effect, consider the discrete signal $x[n]$ described in (10) with $A_{0}=-50 \sqrt{3}$, $A_{1}=100, \varphi_{1}=30^{\circ}, N=32$ and $\tau=50 \mathrm{~ms}$.

Figs. 2(a) and 2(b) show the estimated amplitude of $\bar{X}_{1}[n]$ and $\bar{X}[n]$ computed via the HCDFT and FCDFT algorithms, respectively. As can be seen, the decaying dc component jeopardizes the performance of both algorithms, but noticeably the HCDFT is significantly more affected, resulting very poor transient response and unaccepted errors in phasor estimation. Notwithstanding, regarding the results for $\bar{X}_{1}[n]$, one can see that the HCDFT algorithm is two times faster than the FCDFT algorithm. Therefore, the HCDFT algorithm may be applied along with efficient decaying dc component removal strategy to provide high speed protective relaying, as long as it is guaranteed that no even harmonics could take place.

\section{Proposed Modified DFT-Based Algorithms}

Since the proposed modification can be applied to both conventional FCDFT and HCDFT algorithms interchangeably (i.e, it only depends on the value of the data window length $M$ ), a generalized formulation is presented hereafter.

Aiming to describe the proposed modified DFT-based algorithms, for the sake of simplicity, consider the discrete fault signal $x[n]$ described in (10), which has only one decaying dc component. The Appendix presents how they can deal with multiple decaying dc components.
The outputs of the conventional DFT-based real and imaginary part filters to the most recent data window can be computed substituting (10) in (2) and (3), respectively:

$$
\begin{aligned}
X_{r e}[n]= & \underbrace{\frac{2}{M} \sum_{m=1}^{M} A_{0} \Gamma^{n-M+m} \cos (m \delta)}_{X_{r e, d c}[n]} \\
+ & \underbrace{\frac{2}{M} \sum_{m=1}^{M} A_{1} \cos [(n-M+m) \delta}_{X_{r e 1}[n]}+\varphi_{1}] \cos (m \delta) \\
= & \underbrace{-\frac{2}{M} \sum_{m=1}^{M} A_{0} \Gamma^{n-M+m} \sin (m \delta)}_{X_{i m}[n]=} \\
= & -\underbrace{\frac{2}{M} \sum_{m=1}^{M} A_{1} \cos \left[\left(n-M+X_{r e 1}[n]\right.\right.}_{X_{i m, d c}[n]} \\
X_{i m, d c}[n]+X_{i m 1}[n] &
\end{aligned}
$$

where $X_{r e 1}[n]$ and $X_{i m 1}[n]$ are, respectively, the real and imaginary parts of the accurate fundamental frequency phasor $\bar{X}_{1}[n]$, which does not include the decaying dc component effect; $X_{r e, d c}[n]$ and $X_{i m, d c}[n]$ represent, respectively, the decaying dc component effect in the outputs of the real and imaginary part filters.

Similarly, the real and imaginary part filters applied to the one sample delayed data window yield:

$$
\begin{aligned}
& X_{r e}[n-1]=\lambda X_{r e, d c}[n]+\cos \delta X_{r e 1}[n]+\sin \delta X_{i m 1}[n], \\
& X_{i m}[n-1]=\lambda X_{i m, d c}[n]-\sin \delta X_{r e 1}[n]+\cos \delta X_{i m 1}[n],
\end{aligned}
$$

where $\lambda=\Gamma^{-1}$.

Substituting (16) and (17) in (18) and (19) yields:

$$
\begin{array}{r}
(\cos \delta-\lambda) X_{r e 1}[n]+\sin \delta X_{i m 1}[n] \\
=X_{r e}[n-1]-\lambda X_{r e}[n] \\
-\sin \delta X_{r e 1}[n]+(\cos \delta-\lambda) X_{i m 1}[n] \\
=X_{i m}[n-1]-\lambda X_{i m}[n]
\end{array}
$$

From (20) and (21), $X_{r e 1}(k)$ and $X_{i m 1}(k)$ is obtained as:

$$
\begin{aligned}
& X_{r e 1}[n]=a\left\{X_{r e}[n-1]-\lambda X_{r e}[n]\right\} \\
& -b\left\{X_{i m}[n-1]-\lambda X_{i m}[n]\right\} \\
& X_{i m 1}[n]=b\left\{X_{r e}[n-1]-\lambda X_{r e}[n]\right\} \\
& +a\left\{X_{i m}[n-1]-\lambda X_{i m}[n]\right\}
\end{aligned}
$$

where $a=\frac{\cos \delta-\lambda}{c}, b=\frac{\sin \delta}{c}$ and $c=\lambda^{2}-2 \cos \delta \lambda+1$.

By the analysis of (22) and (23), it can be seen that $\lambda$ is necessary to obtain $X_{r e 1}[n]$ and $X_{i m 1}[n]$. To do so, different algorithms reported in the literature on the subject can be used. However, a new strategy is proposed here, as discussed next. 


\section{A. On Estimating $\lambda$}

Consider that the real part filter is applied to the evensample-set of the most recent data window. Therefore, substituting (10) in (5) results:

$$
\begin{aligned}
X_{r e}^{\text {even }}[n] & =\underbrace{\frac{2}{M} \sum_{m=1}^{M / 2} A_{0} \Gamma^{n-M+2 m} \cos (2 m \delta)}_{X_{r e, d c}^{\text {even }}[n]} \\
+ & \underbrace{\frac{2}{M} \sum_{m=1}^{M / 2} A_{1} \cos \left[(n-M+2 m) \delta+\varphi_{1}\right] \cos (2 m \delta)}_{\frac{1}{2} X_{r e 1}[n]} \\
& =X_{r e, d c}^{\text {even }}[n]+\frac{1}{2} X_{r e 1}[n]
\end{aligned}
$$

where $X_{r e, d c}^{\text {even }}[n]$ represents the decaying dc component effect in the output of the real part filter applied to the even-sampleset of the most recent data window.

It can be verified that the real part filter applied to the evensample-set of the one sample delayed data window results:

$$
\begin{aligned}
X_{r e}^{\text {even }}[n-1]= & \lambda X_{r e, d c}^{\text {even }}[n] \\
& +\frac{1}{2}\left\{\cos \delta X_{r e 1}[n]+\sin \delta X_{i m 1}[n]\right\}
\end{aligned}
$$

Substituting (24) in (25) yields:

$$
\begin{aligned}
(\cos \delta-\lambda) X_{r e 1}[n] & +\sin \delta X_{i m 1}[n]= \\
& 2\left\{X_{r e}^{\text {even }}[n-1]-\lambda X_{r e}^{\text {even }}[n]\right\}
\end{aligned}
$$

From (20) and (26), $\lambda$ can be estimated as follows:

$$
\lambda=\frac{X_{r e}[n-1]-2 X_{r e}^{\text {even }}[n-1]}{X_{r e}[n]-2 X_{r e}^{\text {even }}[n]}
$$

As it can be seen, the proposed strategy requires $M+1$ samples to correct estimate $\lambda$. Aiming to provide soft convergence to the actual value, a moving average process can be performed, by using a set of few successive estimations of $\lambda$.

\section{B. On Implementing the Proposed Algorithms}

The flowchart diagram shown in Fig. 3 summarizes the overall process to implement proposed algorithms. In order to do so, some modules readily available in numerical relays are used, so that their description are not provided here. The anti-aliasing filtering is applied prior to the signal sampling. The frequency estimation module provides the fundamental frequency of the system to properly adapt the sampling rate during off-nominal frequency operation, thereby the analogto-digital (A/D) conversion is carried out. Thereafter, the proposed phasor estimation algorithms can be performed. The difference between them lies in the fact $M=N$ for the proposed modified FCDFT (MFCDFT) and $M=N / 2$ for the proposed modified HCDFT (MHCDFT), where $M$ is the data window length. Then, the imaginary part filter $h_{i m}$ (i.e., the sine filter) is applied to the data window of $x[n]$, thereby $X_{i m}[n]$ is computed using (3). In order to compute $X_{r e}[n]$, the real part filter $h_{r e}$ (i.e., the cosine filter) is applied to both

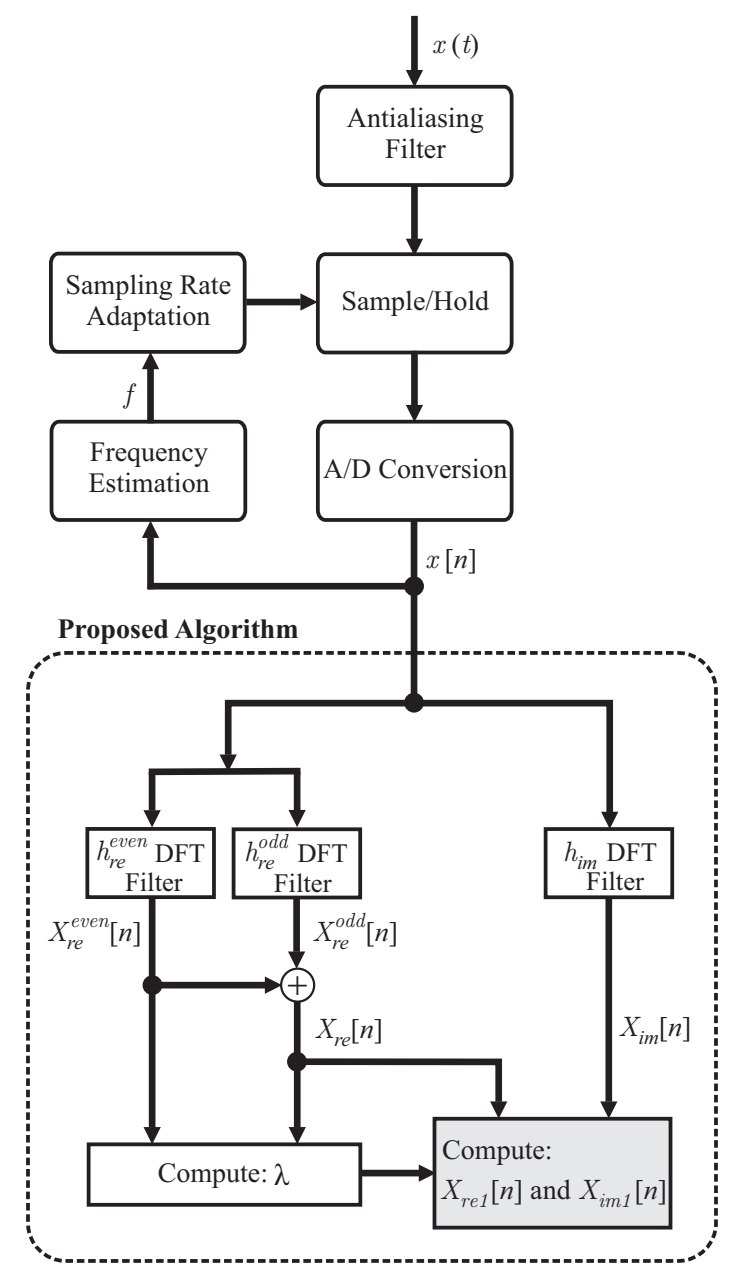

Fig. 3. Flowchart diagram of proposed algorithms.

even- and odd-samples-sets within the data window of $x[n]$, as described in (4)-(6). The parameter $\lambda$ is then estimated by means of (27). Finally, the real part $X_{r e 1}[n]$ and the imaginary part $X_{i m 1}[n]$ of the accurate fundamental frequency phasor is obtained without the decaying dc component effect using $X_{r e}[n], X_{i m}[n]$ and $\lambda$, as described in (22) and (23).

\section{PERFormance EVAluation}

The performance of the proposed algorithms were compared with the ones of following DFT-based phasor estimation algorithms: Guo et al. [14], Kang et al. [16] and Zadeh et al. [18]; as well as with the one of the Least Error Square (LES) algorithm [22]. Aiming to do so, both computer-simulated and ATP-generated signals were evaluated. In both cases, simulations were carried out using time step of 1.041667 $\mu$ s (16000 samples within one cycle of $60 \mathrm{~Hz}$ ), emulating analog signals. Then, a second order low-pass anti-aliasing Butterworth filter with cutoff frequency at $240 \mathrm{~Hz}$ was applied. By discarding samples, the filtered signals were resampled to 16 samples per cycle of $60 \mathrm{~Hz}$ (i.e., using a sampling frequency $f_{s}=960 \mathrm{~Hz}$ ) to evaluate time response of the algorithms. The obtained results are discussed next. 


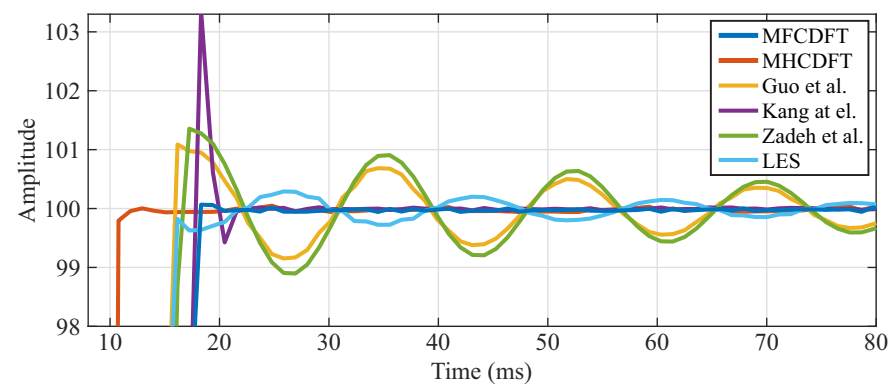

(a)

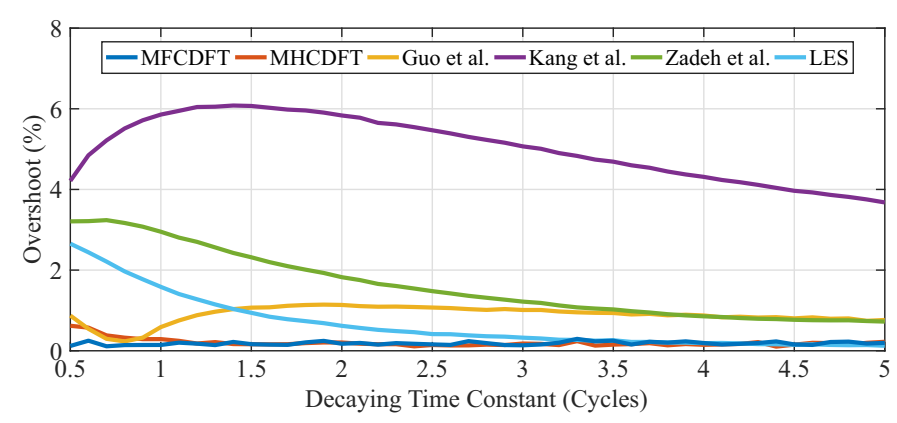

(b)

Fig. 4. The performance of the evaluated algorithms applied to the test signal (28) with: (a) $\tau_{A}=3$ cycles; (b) $\tau_{A}$ ranging from 0.5 to 5.0 cycles [2].

\section{A. On Evaluating Computer-Simulated Signals}

The performance of the algorithms were evaluated using the following test signal:

$$
x[n]=A_{0} \Gamma_{A}^{n}+B_{0} \Gamma_{B}^{n}+A_{1} \cos \left(n \delta+\varphi_{1}\right)+w[n],
$$

where $\Gamma_{A}=e^{-\Delta t / \tau_{A}}$, with $\tau_{A}$ varying; $\Gamma_{B}=e^{-\Delta t / \tau_{B}}$, with $\tau_{B}=20$ cycles (the secondary decaying dc time constant); $A_{0}=90, B_{0}=10, A_{1}=-100$ and $\varphi_{1}=150^{\circ}$; and $w[n]$ is a white random noise with $S N R=50 \mathrm{~dB}$. Here, $\tau_{A}$ represents the decaying dc time constant of the primary current, whereas $\tau_{B}$ represents the dc decaying time constant that arises due to the secondary circuit of the current transformer (CT).

In Fig. 4(a), it is shown the performance of the evaluated algorithms applied to the test signal described in (28) with $\tau_{A}=3$ cycles. It can be seen that proposed algorithms lead to the smallest overshoot and settling time in phasor estimation, even taking into account noise effect. Moreover, these results reveal that proposed algorithms are able to properly deal with the effect of both primary and secondary decaying dc components, as discussed in Appendix.

In Fig. 4(b), it is shown the performance of the evaluated algorithm in respect to the percentage of overshoot for the fundamental phasor amplitude estimation, considering the test signal with $\tau_{A}$ raging from 0.5 to 5 cycles [2]. One can verify that proposed algorithms present the best performance, leading to almost zero overshoot for a wide range of $\tau_{A}$, even taking into account noise effect.

\section{B. On Evaluating ATP-Generated Signals}

The basic system model shown in Fig. 5 was used to generate fault data to evaluate the performance of the algorithms. It is a $230 \mathrm{kV} / 60 \mathrm{~Hz}$ ATP power system reference model proposed by the IEEE Power System Relaying Committee.

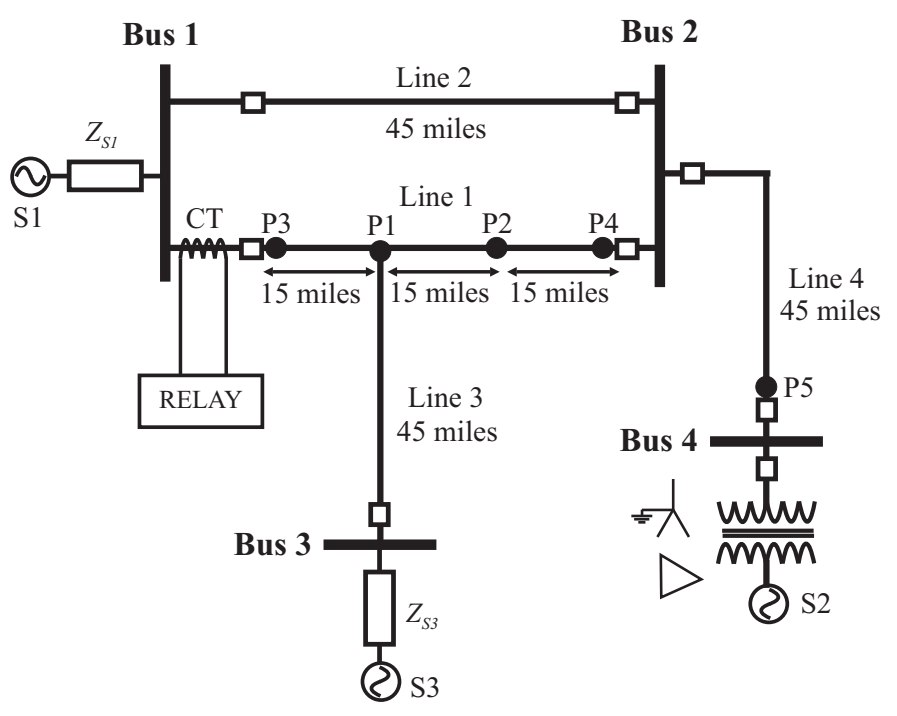

Fig. 5. $230 \mathrm{kV}$ power system single line diagram [23].

In this model, there are two ideal sources $\mathrm{S} 1$ and $\mathrm{S} 3$ and a synchronous machine $\mathrm{S} 2$, which is connected to bus 4 through a grounded Y- $\Delta$ two-winding transformer. The transmission lines consist of one pair of mutually coupled lines (between buses 1 and 2), out of which one is a three terminal line connected to bus 3 , and a single line connected between bus 2 and bus 4. The CT shown in Fig. 5 is also included in the system model. A thorough description of this reference system model and its .atp files are available in [23]. The results shown next represent the currents measured at the secondary of the CT model, thereby both primary and secondary decaying dc component effects are taken into account.

1) Faults Without CT Saturation: The performance of the evaluated algorithms for faults with no $\mathrm{CT}$ saturation are illustrated in Fig. 6(a) and Fig. 6(b).

In Fig. 6(a), it is shown the amplitude of phase B current for a bolted single-line-to-ground fault in phase B at point P1, whereas the amplitude of phase $\mathrm{C}$ current for a bolted doubleline-to-ground fault between phase $\mathrm{A}$ and $\mathrm{C}$ at point $\mathrm{P} 2$ is shown in Fig. 6(b). In both cases, it can be seen that proposed algorithms lead to small overshoot and provide quite fast settling time. Moreover, they also present good performance during transient interval, within which data windows contain both pre-fault and fault samples. As a result, proposed algorithms may simultaneously improve the speed and accuracy of protection function.

2) Faults With CT Saturation: The CT core saturation still requires major concerns in power system protection. Actually, the secondary current of saturated CTs is quite distorted, presenting large harmonic content and reduced fundamental component. As a result, protection functions may misoperate. Therefore, it is important to ensure that phasor estimation algorithms perform properly during $\mathrm{CT}$ saturation.

In Fig. 7(a), it is depicted the secondary phase A current of the CT model shown in Fig. 5 for a close-in three-phase internal fault at point P3. In this case, the CT model reported in [23] originally does not saturate. Therefore, the CT secondary burden was increased to force its core saturation. 


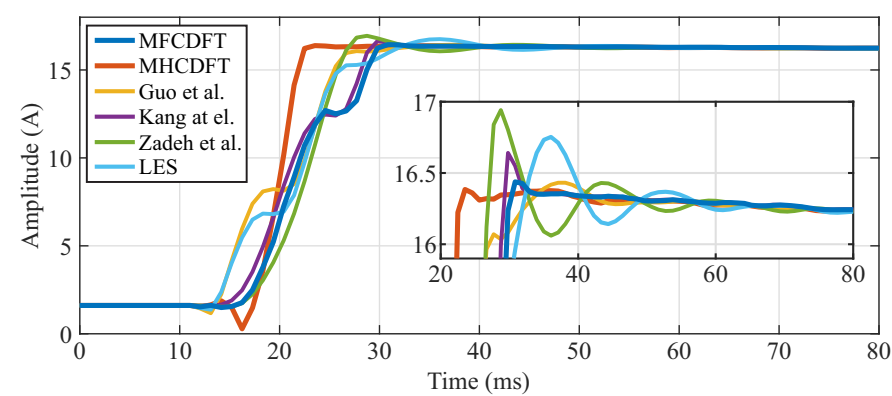

(a)

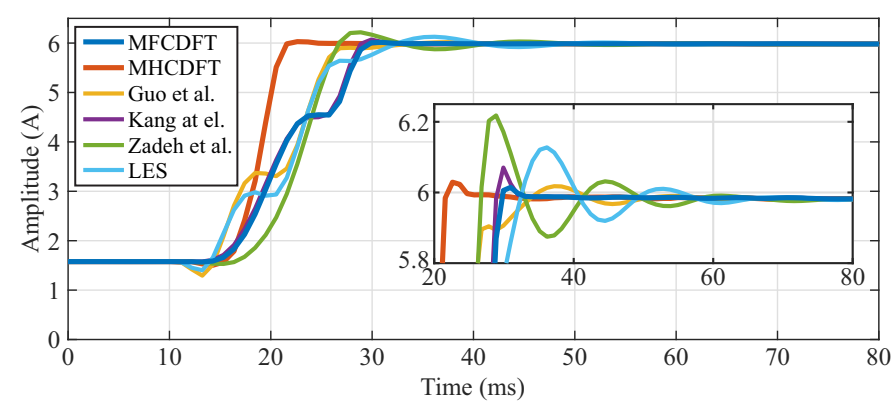

(b)

Fig. 6. The performance of the evaluated algorithms on estimating the amplitude of: (a) phase B current for a BG bolted fault at point P1; (b) phase $\mathrm{C}$ current for a CAG bolted fault at point $\mathrm{P} 2$.

In Fig. 7(b), it is presented the performance of the evaluated algorithms when applied to the distorted CT secondary current shown in 7(a). As one can see, the proposed MFCDFT algorithm performs similarly to other full-cycle DFT-based algorithms, since they reject all harmonic components. Conversely, the proposed MHCDFT algorithm presents quite poor response, since it does not filter out even harmonics of the distorted secondary current. However, it is noteworthy to point out that it is a characteristic inherited from the conventional HCDFT filters (see Section II-B). Therefore, one ought to bear in mind that the MHCDFT algorithm cannot be used whenever even harmonics could take place. In fact, half-cycle filters have been used in real world applications of EHV and UHV transmission lines protection, where high-speed tripping is required and CT saturation is rare [24].

3) Power Swings: Power swing is a phenomenon of oscillation in machine rotor angle often caused by disturbances, such as transmission line tripping, loss of generation and load rejection [25]. It causes modulation of voltage and current signals due to low-frequency components, causing oscillations on amplitude and phase of their phasors [26].

In order to analyze the performance of the evaluated algorithms for power swings, it was simulated a permanent threephase bolted fault at point P5. In Fig. 8(a), it is depicted the phase A current in the secondary of the CT model, revealing that power system underwent an unstable power swing [25]. The performance of the evaluated algorithm are shown in Fig. 8(b). It can be seen that they perform quite similar. In fact, even though damped oscillation modes take place during power swing, the pure decaying dc component is negligible for this kind of phenomena, such that different strategies to eliminate the decaying dc component have no effect and the filtering characteristics of the conventional DFT filters prevail.

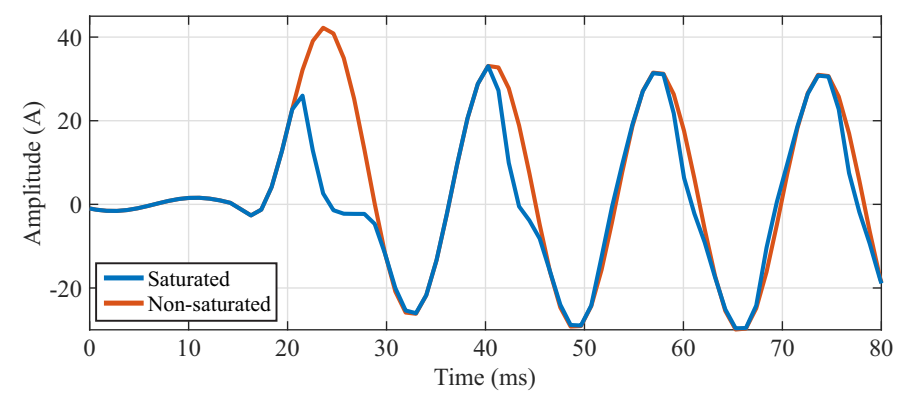

(a)

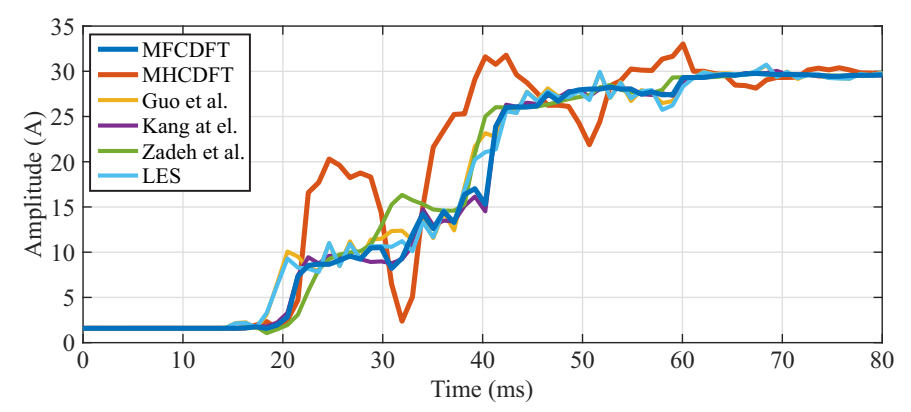

(b)

Fig. 7. The performance of the evaluated algorithms during CT saturation: (a) instantaneous current; (b) phasor amplitude estimation.

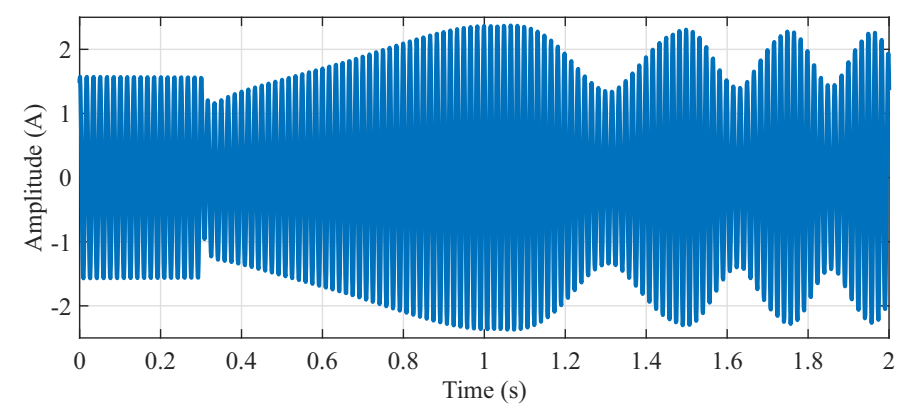

(a)

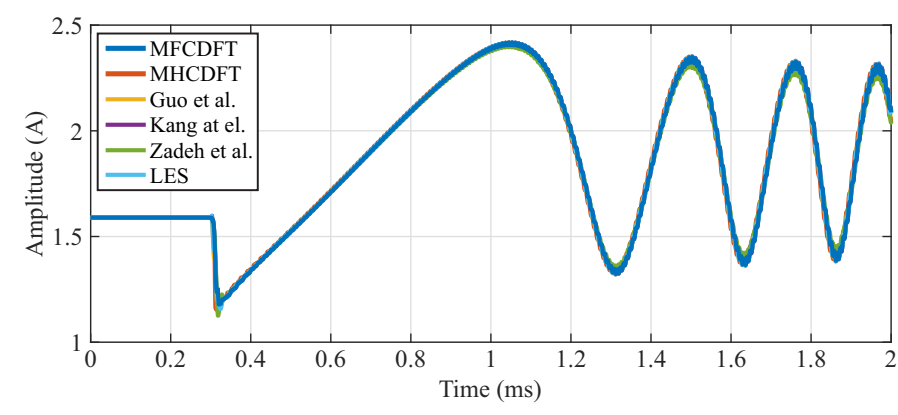

(b)

Fig. 8. The performance of the evaluated algorithms during power swing: (a) instantaneous current; (b) phasor amplitude estimation.

\section{Massive Fault Data Analysis}

Aiming to further analyze the performance of evaluated algorithms, a massive data analysis was performed. In order to do so, extensive simulations of faults in the power system model shown in Fig. 5 were carried out, by means of the variation of location, resistance, inception time and type of faults, in accordance with the Tab. I. As a whole, the variation of these parameters resulted in a total of 1800 faults. 
TABLE I

PARAMETERS USED TO SIMULATE THE MASSIVE FAULT DATA.

\begin{tabular}{cc}
\hline Parameter & Chosen Values \\
\hline \hline Fault location & Points P1, P2, P3 and P4 \\
\hline Fault resistance $(\Omega)$ & $\begin{array}{c}\text { Phase-Phase: } 0,5,10, \cdots, 15 \text { and } 20 \\
\text { Phase-Ground: } 0,25,50,75 \text { and } 100\end{array}$ \\
\hline Fault inception $(\mathrm{ms})$ & $70,71,72,73, \cdots, 84,85$ and 86 \\
\hline Fault type & AG-BG-CG-AB-AC-BC \\
& ABG-ACG-BCG-ABC \\
\hline
\end{tabular}

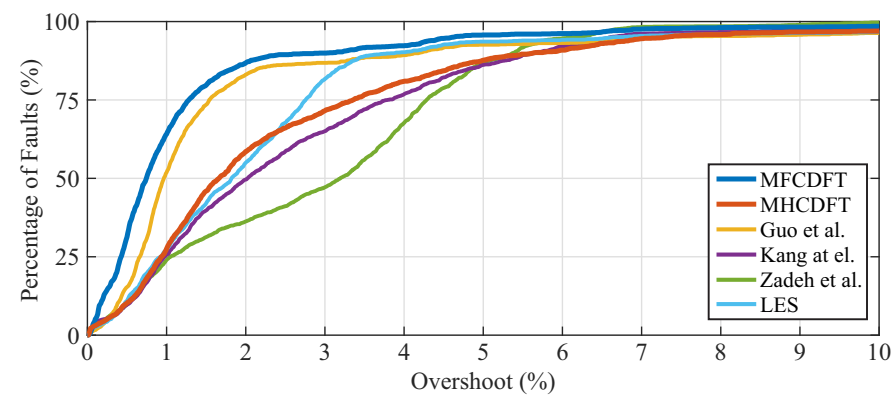

Fig. 9. The cumulative frequency polygon plotting the percentage of faults versus the maximum overshoot in phasor estimation.

In Fig. 9, it is shown a cumulative frequency polygon that plots the percentage of faults versus the maximum overshoot in phasor estimation. As it can be seen, the proposed MFCDFT presents the best performance, providing the smallest overshoot for the most of faults. The proposed MHCDFT, in turn, presents a performance a little bit worse, but that is similar to the one obtained by the algorithm reported by Kang et al. [16]. For example, the proposed MFCDFT provides an overshoot equal to or smaller than $1 \%$ for about $62 \%$ of the faults, whereas for the algorithm reported by Guo et al. [14] this percentage is about $50 \%$, and for the remaining algorithms is about $25 \%$ only.

\section{Discussion}

By the overall analysis of the proposed formulation and obtained results, the following findings arise:

- Among the different strategies to deal with fundamental frequency deviation, the variable sampling measurement environment that is widely used in numerical relays has been considered. Therefore, by doing so, the proposed DFT-based algorithms can be used in off-nominal fundamental frequency scenarios straightforwardly.

- It is well-known that multiple decaying dc components may take place in current signals: one or more caused by the primary power systems, depending on the system topology (different infeed currents) and the system nonhomogeneity (different $X / R$ ratios); one caused by the secondary CT circuitry [3]; one or more caused by the transient response of anti-aliasing filters [12]; etc. Despite the proposed formulation has cover one decaying dc component only, the piecewise approximation described in Appendix shows how the proposed algorithms can overcome this drawback.
- Even though the idea of splitting the DFT formulas to real and imaginary parts and then to odd and even sub-parts has already been reported [16], [17], it is noteworthy to point out that the performance of the proposed algorithms are quite different from those ones, revealing they are not just a different way of combining the outputs of DFT filters, but they are simple and efficient way of doing it.

- It is noteworthy to point out that during the steady state the different strategies to eliminate the decaying dc component have no effect, such that different algorithms have almost the same performance. Therefore, in order to ensure the secure and reliable operation of protection relays, the accuracy of phasor estimation algorithms must be compared through their transient response.

\section{Conclusions}

This paper presents a generalized modification of the conventional FCDFT and HCDFT phasor estimation algorithms, thereby the decaying dc component effect on phasor estimation is gotten rid of. The obtained results reveal that the proposed modified algorithms lead to phasor estimation with smooth transient response, minimal overshoot and fast settling time. Moreover, proposed algorithms are able to deal with multiple decaying dc components. Accordingly, one can suppose that proposed algorithms can be successfully used in numerical digital relaying applications.

\section{REFERENCES}

[1] A. G. Phadke and J. S. Thorp, Computer Relaying for Power Systems, 2nd ed. West Sussex, England: John Wiley \& Sons, 2009.

[2] E. O. Schweitzer and D. Hou, "Filtering for protective relays," Schweitzer Engineering Laboratories, Pullman, USA, Tech. Rep., 1993.

[3] S. H. Horowitz, A. G. Phadke, and J. K. Niemira, Power System Relaying, 2nd ed. West Sussex, England: John Wiley \& Sons, 2014.

[4] D. G. Hart, D. Novosel, and R. A. Smith, "Modified cosine filters," U.S. Patent 6,154,687, Nov. 2000.

[5] E. Rosolowski, J. Iżykowski, and B. Kasztenny, "Adaptive measuring algorithm suppressing a decaying dc component for digital protective relays," Electric Power Systems Research, vol. 60, pp. 99-105, Sep. 2001.

[6] T. S. Sidhu, X. Zhang, and V. Balamourougan, "A new half-cycle phasor estimation algorithm," IEEE Transactions on Power Delivery, vol. 20, no. 2, pp. 1299-1305, Apr. 2005.

[7] Y.-S. Cho, C.-K. Lee, G. Jang, and H. J. Lee, "An innovative decaying dc component estimation algorithm for digital relaying," IEEE Transactions on Power Delivery, vol. 24, no. 1, pp. 73-78, Jan. 2009.

[8] K. M. Silva and B. F. Küsel, "On combining wavelet-based designed filters and an adaptive mimic filter for phasor estimation in digital relaying," Electric Power Systems Research, vol. 92, pp. 60-72, Nov. 2012.

[9] Z. Jiang, S. Miao, and P. Liu, "A modified empirical mode decomposition filtering-based adaptive phasor estimation algorithm for removal of exponentially decaying dc offset," IEEE Transactions on Power Delivery, vol. 29, no. 3, pp. 1326-1334, Jun. 2014.

[10] S. A. Gopalan, Y. Mishra, V. Sreeram, and H. H.-C. Iu, "An improved algorithm to remove dc offsets from fault current signals," Accepted for future publication in IEEE Transactions on Power Delivery, 2016.

[11] J.-C. Gu and S.-L. Yu, "Removal of dc offset in current and voltage signals using a novel fourier filter algorithm," IEEE Transactions on Power Delivery, vol. 15, no. 1, pp. 73-79, Jan. 2000.

[12] S.-L. Yu and J.-C. Gu, "Removal of decaying dc in current and voltages signals using a mofied fourier filter algorithm," IEEE Transactions on Power Delivery, vol. 16, no. 3, pp. 372-379, Jul. 2001.

[13] T. S. Sidhu, X. Zhang, F. Albasri, and M. S. Sachdev, "Discretefourier-transform-based technique for removal of decaying dc from phasor estimates," IEE Proceedings in Generation, Transmission and Distribution, vol. 150, no. 6, pp. 745-752, Nov. 2003. 
[14] Y. Guo, M. Kezunovic, and D. Chen, "Simplified algorithms for removal of the effect of exponentially decaying dc-offset on the fourier algorithm," IEEE Transactions on Power Delivery, vol. 18, no. 3, pp. 711-717, Jul. 2003.

[15] C.-S. Chen, C.-W. Liu, and J.-A. Jiang, "Application of combined adaptive fourier filtering technique and fault detector to fast distance protection," IEEE Transactions on Power Delivery, vol. 21, no. 2, pp. 619-626, Apr. 2006.

[16] S.-H. Kang, D.-G. Lee, S.-R. Nam, P. A. Crossley, and Y.-C. Kang, "Fourier transform-based modified phasor estimation method immune to the effect of the dc offsets," IEEE Transactions on Power Delivery, vol. 24, no. 3, pp. 1104-1111, Jul. 2009.

[17] K. M. Silva and B. F. Küsel, "Dft based phasor estimation algorithm for numerical digital relaying," Electronics Letters, vol. 49, no. 6, 2013.

[18] M. R. D. Zadeh and Z. Zhang, "A new dft-based current phasor estimation for numerical protective relaying," IEEE Transactions on Power Delivery, vol. 28, no. 4, pp. 2172-2179, Oct. 2013.

[19] A. Rahmati and R. Adhami, "An accurate filtering technique to mitigate transient decaying dc offset," IEEE Transactions on Power Delivery, vol. 29, no. 2, pp. 966-968, Apr. 2014.

[20] A. Phadke and J. Thorp, Synchronized Phasor Measurements and Their Applications, 1st ed. New York, USA: Springer, 2008.

[21] A. V. Oppenheim and R. W. Schafer, Discrete-Time Signal Processing. USA: Pearson, 2009.

[22] Understanding Microprocessor-based Technology Applied to Relaying, IEEE Power System Relaying Committee WG I-01, Jan. 2009. Available: http://www.pes-psrc.org.

[23] EMTP Reference Models for Transmission Line Relay Testing, IEEE Power System Relaying Committee WG D10, Aug. 2004. Available: http://www.pes-psrc.org.

[24] A. Guzmán, J. Mooney, G. Benmouyal, and N. Fischer, "Transmission line protection system for increasing power system requirements," in 55th Annual Conference for Protective Relay Engineers, College Station, US, Apr. 2002.

[25] Power swing and out-of-step considerations on transmission line, IEEE Power System Relaying Committee WG D6, Jul. 2005. Available: http://www.pes-psrc.org.

[26] J. G. Rao and A. K. Pradhan, "Accurate phasor estimation during power swing," IEEE Transactions on Power Delivery, vol. 31, no. 1, pp. 130137, Feb. 2016.

\section{APPENDIX}

This appendix presents how the proposed algorithms deal with two decaying dc components as a piecewise approximated dc offset. Indeed, one can verify that the justification presented here generalizes the one reported in [16], since it can be applied to both HCDFT and FCDFT conventional algorithms interchangeably, by choosing the data window length $M$ properly (see Section II-A).

Consider a discrete signal $x[n]$ with two decaying $\mathrm{dc}$ and a pure co-sinusoidal components as follows:

$$
x[n]=A \cos (n \delta+\varphi)+B \Gamma_{B}^{n}+C \Gamma_{C}^{n}
$$

where $\Gamma_{B}=e^{-\Delta t / \tau_{B}}$ and $\Gamma_{C}=e^{-\Delta t / \tau_{C}}$.

Applying the complex form of DFT-based algorithms yields:

$$
\bar{X}[n]=\bar{X}_{1}[n]+\bar{X}_{d c, B}[n]+\bar{X}_{d c, C}[n],
$$

where $\bar{X}_{1}[n]=A e^{j \varphi+n \delta}$ is the desired fundamental frequency phasor, whereas $\bar{X}_{d c, B}[n]$ and $\bar{X}_{d c, C}[n]$ are the outputs of the DFT-based algorithms for the decaying dc components, which are computed as follows (see Section II-C):

$$
\begin{aligned}
& \bar{X}_{d c, B}[n]=\frac{2}{M} B \Gamma_{B}^{n-M} \frac{\left(1-\Gamma_{B}^{M}\right)}{1-\Gamma_{B} e^{-j \delta}} \\
& \bar{X}_{d c, C}[n]=\frac{2}{M} C \Gamma_{C}^{n-M} \frac{\left(1-\Gamma_{C}^{M}\right)}{1-\Gamma_{C} e^{-j \delta}}
\end{aligned}
$$

One can prove that there is an equivalent decaying $\mathrm{dc}$ component $x_{d c, E}[n]=E \Gamma_{E}^{n}$ that approximates the combined effect of the two decaying dc components of $x[n]$, so that:

$$
\begin{aligned}
E \Gamma_{E}^{n-M} \frac{\left(1-\Gamma_{E}^{M}\right)}{1-\Gamma_{E} e^{-j \delta}}= & B \Gamma_{B}^{n-M} \frac{\left(1-\Gamma_{B}^{M}\right)}{1-\Gamma_{B} e^{-j \delta}} \\
& +C \Gamma_{C}^{n-M} \frac{\left(1-\Gamma_{C}^{M}\right)}{1-\Gamma_{C} e^{-j \delta}}
\end{aligned}
$$

Decomposing (A.5) into real and imaginary part yields:

$$
\begin{gathered}
\frac{E \Gamma_{E}^{n-M}\left(1-\Gamma_{E}^{M}\right)}{1-2 \Gamma_{E} \cos \delta+\Gamma_{E}^{2}}\left(1-\Gamma_{E} \cos \delta\right)=B_{r e}+C_{r e} \\
\frac{E \Gamma_{E}^{n-M}\left(1-\Gamma_{E}^{M}\right)}{1-2 \Gamma_{E} \cos \delta+\Gamma_{E}^{2}} \Gamma_{E} \sin \delta=B_{i m}+C_{i m}
\end{gathered}
$$

where

$$
\begin{aligned}
B_{r e} & =\frac{B \Gamma_{B}^{n-M}\left(1-\Gamma_{B}^{M}\right)}{1-2 \Gamma_{B} \cos \delta+\Gamma_{B}^{2}}\left(1-\Gamma_{B} \cos \delta\right) \\
B_{i m} & =\frac{B \Gamma_{B}^{n-M}\left(1-\Gamma_{B}^{M}\right)}{1-2 \Gamma_{B} \cos \delta+\Gamma_{B}^{2}} \Gamma_{B} \sin \delta \\
C_{r e} & =\frac{C \Gamma_{C}^{n-M}\left(1-\Gamma_{C}^{M}\right)}{1-2 \Gamma_{C} \cos \delta+\Gamma_{C}^{2}}\left(1-\Gamma_{C} \cos \delta\right) \\
C_{i m} & =\frac{C \Gamma_{C}^{n-M}\left(1-\Gamma_{C}^{M}\right)}{1-2 \Gamma_{C} \cos \delta+\Gamma_{C}^{2}} \Gamma_{C} \sin \delta
\end{aligned}
$$

From (A.6) and (A.7), one can obtain $\Gamma_{E}$ as:

$$
\Gamma_{E}=\frac{B_{i m}+C_{i m}}{\left(B_{i m}+C_{i m}\right) \cos \delta+\left(B_{r e}+C_{r e}\right) \sin \delta}
$$

The result of (A.8) confirms the existence of an equivalent decaying dc component $x_{d c, E}[n]=E \Gamma_{E}^{n}$ that provides a piecewise approximation of the two decaying dc components of $x[n]$. A similar analysis can be performed to reveal that this approximations may also be done to more than two decaying dc components. Therefore, likewise reported in [16], proposed algorithms can properly remove the combined effect of two or more decaying dc components on phasor estimation.

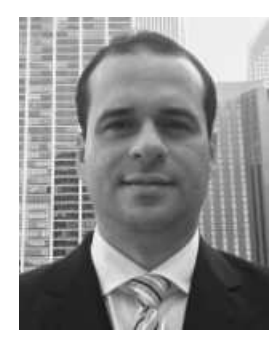

K. M. Silva (M'05) received the B.Sc., M.Sc., and $\mathrm{Ph} . \mathrm{D}$. degrees in electrical engineering from University of Campina Grande (UFCG) in 2004, 2005 , and 2009, respectively. Since 2009, he is Professor at the University of Brasília (UnB) and the head of the Power System Protection Group. His research interests focus on power system protection and electromagnetic transients.

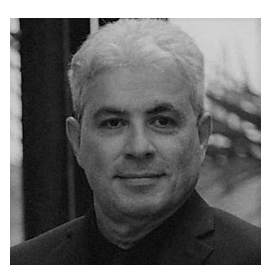

F. A. O. Nascimento received the B.Sc. degree from the University of Brasília (UNB), in 1982, and the M.Sc. and Ph.D. degrees from the Federal University of Rio de Janeiro, in 1985 and 1988, respectively, all in electrical engineering. He is currently a Full Professor at University of Brasilia and the Digital Signal Processing Group coordinator. 